\begin{tabular}{|c|c|}
\hline Citation & $\begin{array}{l}\text { Yang Zhang, Dixian Zhao, Patrick Reynaert (2015), } \\
\text { Millimeter-wave Packaging on Alumina Board for E-band CMOS Power } \\
\text { Amplifiers } \\
\text { IEEE Topical Conference on RF/Microwave Power Amplifiers for Radio and } \\
\text { Wireless Applications (2015) }\end{array}$ \\
\hline Archived version & $\begin{array}{l}\text { Author manuscript: the content is identical to the content of the published } \\
\text { paper, but without the final typesetting by the publisher }\end{array}$ \\
\hline Published version & http://ieeexplore.ieee.org/stamp/stamp.jsp?tp=\&arnumber=7139195 \\
\hline Journal homepage & http://www.radiowirelessweek.org/ \\
\hline Author contact & $\begin{array}{l}\text { Yang.zhang@kuleuven.be } \\
\text { + } 32 \text { (0)16 } 374756\end{array}$ \\
\hline
\end{tabular}




\title{
Millimeter-wave Packaging on Alumina Board for E-band CMOS Power Amplifiers
}

\author{
Yang Zhang, Dixian Zhao and Patrick Reynaert \\ KU Leuven, Dept. Elektrotechniek ESAT-MICAS \\ Kasteelpark Arenberg 10, B-3001, Leuven, Belgium
}

\begin{abstract}
A millimeter-wave bond-wire packaging and micro-strip to waveguide transition are proposed for E-band CMOS power amplifiers. A $700 \mu \mathrm{m}$ cavity is used to shorten bond-wire length, and the bond-wire inductance is compensated by micro-strip matching network, realizing a good packaging performance with $0.3 \mathrm{~dB}$ loss over $30 \mathrm{GHz}$ bandwidth. A low-cost Alumina board is used for micro-strip to waveguide transition, without back-cavity or modification of standard waveguide. Two coupling pads are introduced to realize the transition from 71 to $86 \mathrm{GHz}$ frequency range. Gain, output power and drain efficiency of a 40nm CMOS Eband power amplifier, mounted on the presented package, were measured.
\end{abstract}

Index Terms - package, wire-bond, waveguide, CMOS, E-band, power amplifier, transition.

\section{INTRODUCTION}

Millimeter-wave integrated circuits have become popular recently due to applications such as $60 \mathrm{GHz}$, automotive radar and E-band high speed back-haul links [1]. CMOS technology has been successfully used to implement circuits above $60 \mathrm{GHz}$ [2]. However, characterization and packaging for millimeter-wave integrated circuits remain a challenge, because the wavelength at these high frequencies becomes a fraction of the packaging structures. Measurements through onwafer probing oftentimes give inconsistent results due to damaged probe pads and different probe landing positions. In addition, for radar and E-band high speed back-haul application, on-chip antennas are difficult to implement in CMOS due to the high antenna gain that is typically required in these systems. Therefore, to characterize millimeter-wave integrated circuits and enable low-cost product modules, an easy-to-build, robust and low-cost packaging is needed.

One packaging solution is to build a transition from onchip bond pads to standard waveguides for high frequency signals. Signals go from the chip into micro-strip lines via bond wires or flip-chip bumps, then through a transition into a standard waveguide which are simply screwed on the board. Different types of micro-strip lines to waveguide transitions have been proposed, such as antenna insertion [3], planar probe type [4] and slotcoupled transition [5]. To achieve a wide working bandwidth, mechanical modification of waveguides [4] or

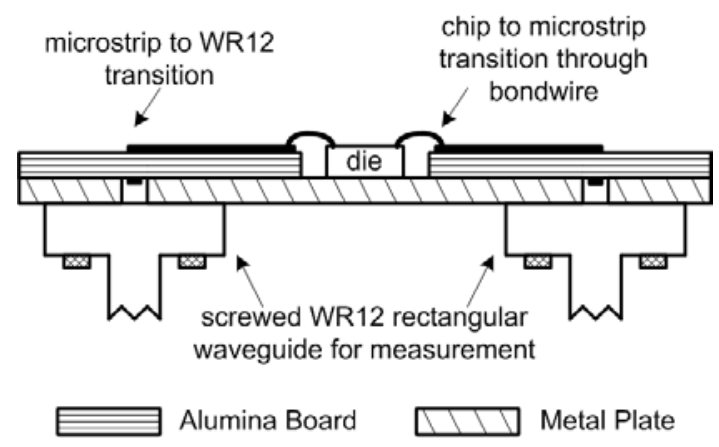

Fig. 1. Cross-section view of the proposed millimeter-wave packaging with two transitions from chip to WR12 waveguide.

a back cavity [6] is usually needed, but these transitions suffer from manufacturing and installation complexity. In this paper, we propose a low-complexity, robust and lowcost packaging solution for an E-band 40nm CMOS power amplifier. Section II gives a detailed explanation of the whole packaging solution design. Section III shows the measured results of a back-to-back micro-strip to waveguide transition, and also the measured results of an E-band CMOS PA using the proposed packaging solution.

\section{Millimeter-WAVE PACKAGING DESIGN}

The proposed packaging solution is implemented on an Alumina board, with a thickness of $254 \mu \mathrm{m}$ and permittivity of 9.5 . Gold wires with diameter of $17 \mu \mathrm{m}$ are used for the bonding. Compared with other substrate materials, alumina board is more robust, low-cost and easy to realize accurate bonding with high adhesion. Fig.1 shows the cross-section view of the proposed packaging which contains two steps: (1) the transition between onchip bond pads and a micro-strip line through bond-wire interconnect and (2) the transition between the micro-strip line to standard WR12 waveguide to launch the required waveguide mode.

\section{A. Chip to Micro-strip transition with Bond-wires}

At millimeter-wave frequencies, the equivalent inductance of bond-wires becomes dominant and influences the performance significantly. To reduce the impact of the interconnect, the length of bond wires in this design is minimized. 


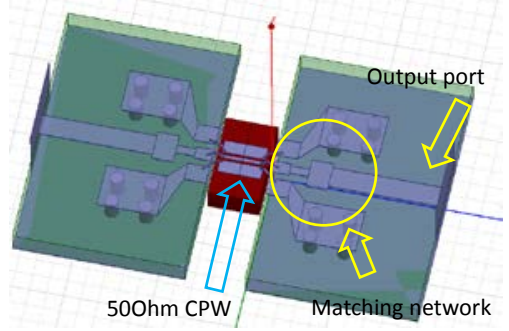

Fig. 2. Back-to-back bond-wire interconnect with cavity, chip is replaced by an on-silicon 50Ohm CPW line.

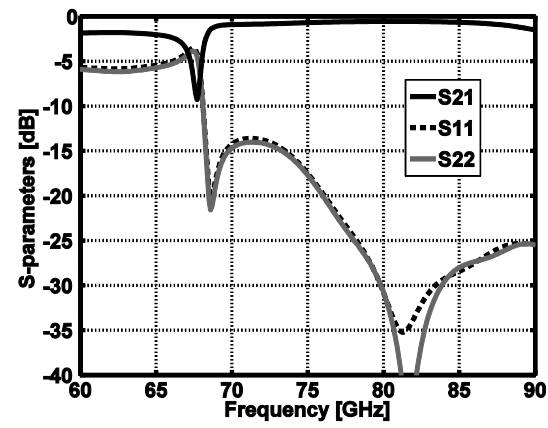

Fig. 3. Simulated S-parameters of back-to-back bond-wire interconnect.

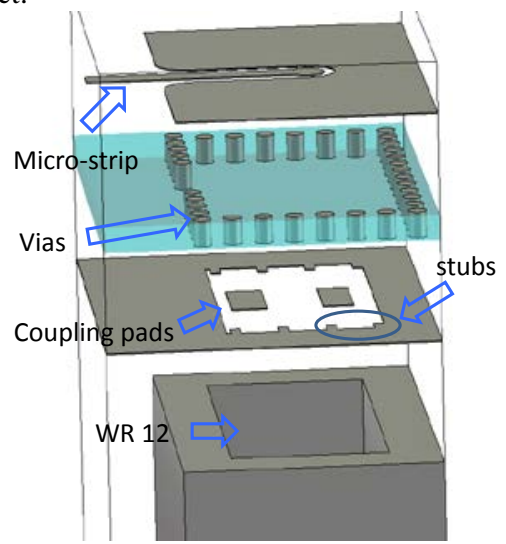

Fig. 4. Micro-strip to WR12 waveguide transition.

Figure 2 shows the details of the proposed bond-wire interconnect. The size of on-chip bond pads is $40 \mu \mathrm{m}$ by $50 \mu \mathrm{m}$ with a pitch of $75 \mu \mathrm{m}$. The chip is placed into a 700 by $1300 \mu \mathrm{m}$ cavity to shorten the bond-wire length, leading to a height difference between chip and board of only $22 \mu \mathrm{m}$. There is a $50 \mu \mathrm{m}$ gap between chip and board, and another $50 \mu \mathrm{m}$ between bond pads and the chip edge. Bonding points on the board is around $35 \mu \mathrm{m}$ from edge, so the total bond wire length of signal path is as low as $240 \mu \mathrm{m}$ and the ground bond wires are slighter longer. During bonding proceeding, the real length of bond wires will be slightly varied.

Micro-strips of different width can be treated as a small inductor or capacitor. As a result, together with bondwires, they are used to form a wideband band-pass filter. The bond-wire inductance is therefore compensated. For

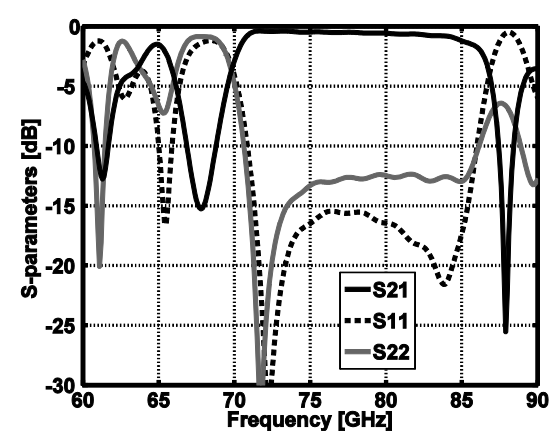

Fig. 5. Simulated S-parameters of micro-strip to WR12 waveguide transition.

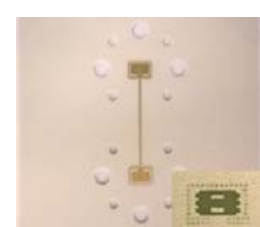

(a)

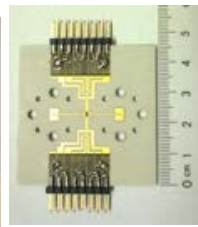

(b)

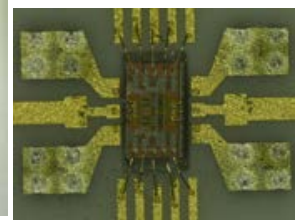

(c)
Fig. 6. (a) back-to-back board with backside view, (b) packaged PA, (c) zoom-in view of the PA die with the bondwire interconnections.

each ground path, four vias are grouped together. As can be seen, a 50Ohm co-planar waveguide (CPW) line on silicon substrate is used to replace the real chip in simulation. Fig. 3 shows the simulated S-parameters of a back-to-back interconnect. Return loss is below -14dB for more than $30 \mathrm{GHz}$ range, and from $71-86 \mathrm{GHz}$ the average insertion loss is $0.6 \mathrm{~dB}$, indicating that one port interconnect has $0.3 \mathrm{~dB}$ loss.

\section{B. Micro-strip to Waveguide Transition}

The Micro-strip to waveguide transition structure is shown in Fig. 4. This work is based on [7]. In order to simplify the implementation, no back-cavity is used, nor the modification of the standard waveguide. The energy is first transformed from the micro-strip mode to the CPW mode, and then coupled to the interface of waveguide by two metal pads at the bottom of the alumina substrate. The width of CPW trace at the end is wider than the other area to get a better coupling. Also, the CPW gap is tuned to reduce radiation loss into outer space. There is a phase difference between these two metal pads, thus yields a strong excitation of TE10 mode into the metal waveguide. To prevent higher transmission mode radiating into the waveguide, some small stubs are placed along the short edges of the interface window. Grounded vias are placed around the transition to shield energy from leakage. Fig. 5 shows the simulated S-parameters of micro-strip to WR12 waveguide transition. The working bandwidth is from 71 to $86 \mathrm{GHz}$, with a return loss lower than $-12 \mathrm{~dB}$ and an average insertion loss of $0.5 \mathrm{~dB}$. 


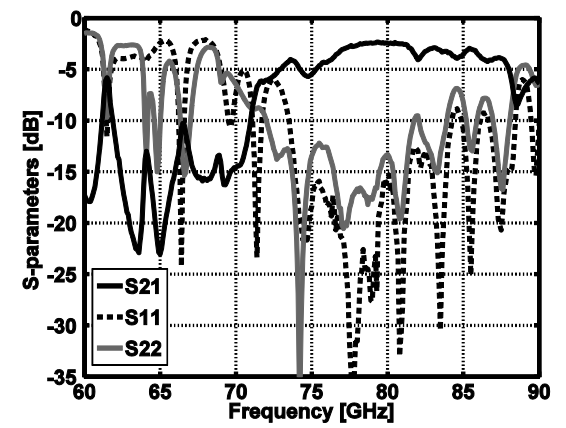

Fig. 7. Measured S-parameters of back-to-back micro-strip to waveguide transition.

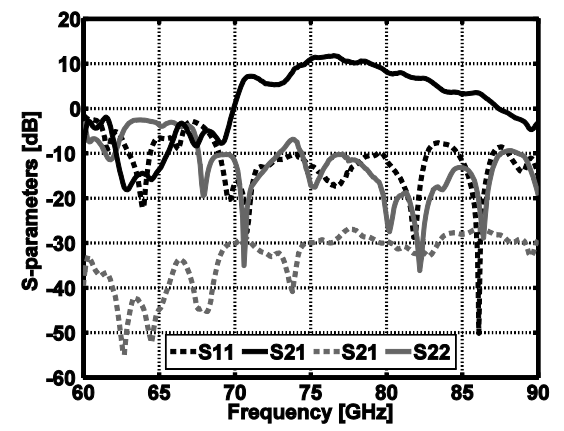

Fig. 8. Measured S-parameters of PA chip with bond-wire packaging and waveguide transition (including packaging loss).

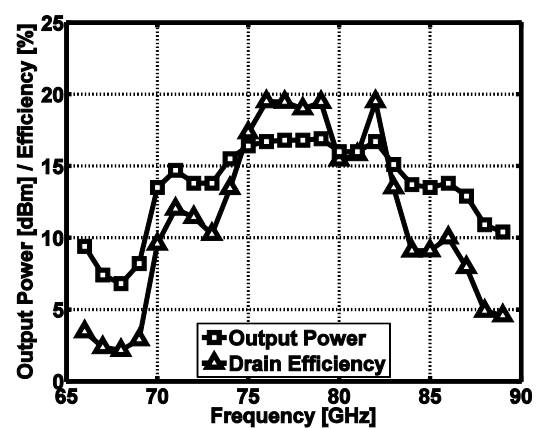

Fig. 9. Measured peak output power and peak drain efficiency curves (including packaging loss).

\section{MEASUREMENT RESULTS}

Figure 6 shows the photos of test boards and packaged PA. The WR-12 waveguide ports from extenders are fixed at the bottom of the Alumina board. The board size is $45 \mathrm{~mm}$ by $45 \mathrm{~mm}$. The total micro-strip length is $20 \mathrm{~mm}$. Fig. 7 shows the measured S-parameters of the back to back micro-strip to waveguide transition. Between $72.8 \mathrm{GHz}$ to $87 \mathrm{GHz}$, the maximum and minimum insertion loss are $5.3 \mathrm{~dB}$ and $2.4 \mathrm{~dB}$ with an average loss of $3.5 \mathrm{~dB}$. The return loss is around $-10 \mathrm{~dB}$ at E-band. The curve is not as flat as expected due to the mechanical fabrication variation which is higher than the specified tolerance.
The E-band PA is designed in a 40nm CMOS technology [1]. Fig. 8 shows the measured performance of the packaged PA chip. The maximum measured gain is $11.7 \mathrm{~dB}$ including all the packaging loss, which is $2 \mathrm{~dB}$ lower than simulation. The measured results can be further improved by better alignment between alumina board and WR12 waveguide. Fig. 9 shows the measured peak output power and drain efficiency of the packaged PA. As can be seen that maximum output power is $16.3 \mathrm{dBm}$ without deembedding the loss and peak drain efficiency is $20 \%$.

\section{CONCLUSION}

A millimeter-wave bond-wire packaging with microstrip to waveguide transition, is proposed for E-band CMOS power amplifiers. The inductance of bond-wires is compensated with micro-strip matching network, resulting in a simulated bandwidth of more than $30 \mathrm{GHz}$ with $0.3 \mathrm{~dB}$ loss. A single layer micro-strip to WR12 waveguide transition is realized. No back-cavity or modification of waveguide is involved. A back to back transition board with $20 \mathrm{~mm}$-long micro-strip measurement shows an average loss of $3.5 \mathrm{~dB}$ within $71-86 \mathrm{GHz}$ range. Gain, peak output power and drain efficiency of an E-band CMOS power amplifier were measured with the proposed set-up.

\section{REFERENCES}

[1] D. Zhao, P. Reynaert, "A 0.9 V 20.9 dBm 22.3\%-PAE Eband power amplifier with broadband parallel-series power combiner in 40nm CMOS”, IEEE International Solid-State Circuits Conference Digest of Technical Papers(ISSCC), pp. 248-289, 2014.

[2] J. Chen, A M. Niknejad, "A compact $1 \mathrm{~V} 18.6 \mathrm{dBm} 60 \mathrm{GHz}$ power amplifier in 65nm CMOS," IEEE International Solid-State Circuits Conference Digest of Technical Papers(ISSCC), pp. 432-433, 2011.

[3] V. Mottonen, A V. Raisanen, "Novel wide-band coplanar waveguide-to-rectangular waveguide transition", IEEE Trans. Microwave Theory Tech., vol. 52, no. 8, pp. 18361842, 2004

[4] E. S. Li, G. X. Tong and D. C. Niu, "Full W-band waveguide-to-microstrip transition with new E-plane probe”, IEEE Microwave and Wireless Components Letters, vol. 23, no. 1, pp. 4-6, 2013.

[5] H. Aliakbarian, S. Radiom, V. Tavakol, et al. "Fully micromachined $\mathrm{W}$-band rectangular waveguide to grounded coplanar waveguide transition”, IET Microwaves, Antennas \& Propagation, vol. 6, pp. 533-540, 2012.

[6] R Y. Fang, C T. Wang, C L. Wang, "A direct CPW-torectangular waveguide transition using a dipole slot antenna”, IEEE Microwave Conference, European. pp. 157160, 2009.

[7] W. Simon, M. Werthen, and I. Wolff, "A novel coplanar transmission line to rectangular waveguide transition", IEEE Microwave Symposium, pp. 257-260, June 1998. 